\title{
Chikungunya adquirida en Recién Nacidos. Reporte de caso
}

\section{Acquired Chikungunya in Newborns:a Case Report}

\author{
Patricia Rolón ${ }^{(1)}$, Rosanna Fonseca ${ }^{(1)}$, Larissa Genes ${ }^{(1)}$, Sonia Pereira ${ }^{(1)}$, Leticia Zapatta ${ }^{(1)}$, Gloria Benítez ${ }^{(2)}$
}

\section{RESUMEN}

Introducción: La fiebre Chikungunya es una enfermedad viral emergente. En las infecciones que ocurren durante el embarazo, el virus no se transmite al feto; el mayor riesgo de transmisión al recién nacido se produce cuando la mujer tiene viremia en el período intraparto, donde la transmisión puede alcanzar alrededor del $49 \%$. Caso Clínico: RN de 16 días de vida procedente de Luque, Paraguay, nacida por parto cesárea, de término sin complicaciones; sin factores de riesgo materno para infecciones; con PN: 3620 gramos, Apgar: 8/9; dada de alta a las 48 hs de vida sin complicaciones. A los 13 días de vida consulta por fiebre y rash, la madre refiere cuadro de 24 horas de evolución de fiebre y rash generalizado acompañado de irritabilidad transitoria; ingresa con el diagnóstico de Sepsis neonatal tardía probable, se inicia ampicilina más cefotaxima, se constató en análisis laboratoriales leucopenia y serología para dengue negativo. En el día 4 de la enfermedad, deterioro progresivo con mayor hipoactividad rechazo alimentario y polipnea, se constata persistencia de leucopenia, y plaquetopenia. Se amplió cobertura antibiótica; requiere intubación orotraqueal; es derivado a Unidad de Cuidados Intensivos Neonatales; donde se observaron las siguientes morbilidades: hepatomegalia, derrame pleural bilateral, crisis convulsiva, hipertensión pulmonar moderada. Buena evolución el día 10 de enfermedad, se procede a extubación programada exitosa, se constató reabsorción total del derrame, ya sin hepatomegalia, con mejoría a nivel de los recuentos de glóbulos blancos, no así en el de las plaquetas que se encontraban en descenso; completó 7 días de antibióticos, cultivos negativos. Al reinterrogatorio se constató nexo epidemiológico. Se recibió serología positiva para Chikungunya IgM, es contra referido al centro de origen con buena evolución, es dado de alta en el día 15 de la enfermedad en buenas condiciones y con recuperación hematológica. Discusión: Este caso es considerado como grave atípico de Chikungunya, el manejo es de sostén para las complicaciones.

Palabras clave: Fiebre Chikungunya, caso atípico, Recién Nacidos.

\section{ABSTRACT}

Introduction: Chikungunya fever is an emerging viral disease.The virus is not transmitted to the fetus when infections occur during pregnancy; the greatest risk of transmission to the newborn occurs when the mother experiences viremia during the intrapartum, when transmission can reach about $49 \%$. Case Report: A 16-day old full-term newborn from Luque, Paraguay, delivered by cesarean section without complications or risk factors for maternal infection, weighing at birth 3620 grams, with an Apgar of 8/9, and discharged at 48 hs of life with no complicationsconsulted on day 13 of life with fever and rash, with the mother reporting 24 hs duration of fever, generalized rash, and transitory irritability, and was hospitalized with a probable diagnosis of late-onset neonatal sepsis, and ampicillin plus cefotaxime initiated; laboratory tests confirmed leukopenia.On day 4 of disease, progressive deterioration was seen with increased hypoactivity, refusal to feed, polypnea, and continued leukopenia and thrombocytopenia. Antibiotic coverage was broadened and orotracheal intubation was required, with the patient transferred to the neonatal intensive care unit, where hepatomegaly, bilateral pleural effusion, seizure, and moderate pulmonary hypertension were observed.Favorable progression was seen on day 10, planned extubation was successful, and complete reabsorption of efflux, resolved hepatomegaly, and improved white blood cell count were found; however, platelet count was seen to be decreasing. On completing day 7 of antibiotic therapy cultures were negative.On reinvestigation, an epidemiological link was found, with positive serology for Chikungunya IgM, and the patient returned to the first treating hospital with favorable progression, and discharged to home on day 15 of disease in good condition with hematological recovery. Discussion: Our patient appears to have had an atypically severe case of Chikungunya, for which management is supportive for complications.

Keywords: Chikungunya fever, atypical; newborns.

1. Unidad de Cuidados Intensivos Neonatales, Hospital Materno Infantil San Pablo. Asunción, Paraguay.

2. Sala de Pediatría, Hospital Regional de Luque. Paraguay.

Correspondencia: Dra. Patricia L. Rolón. E-mail: parox30@gmail.com

http://dx.doi.org/10.18004/ped.2015.abril.42-47

Los autores declaran que no existen conflictos de interés en el presente estudio. 
La fiebre Chikungunya es considerada una enfermedad viral emergente, fue descrita por primera vez durante un brote en el sur de Tanzania en 1952. Desde entonces afecta a millones de personas en el mundo. Es endémica en el sudeste de Asia, África y Oceanía. En la región de las Américas apareció a finales de 2013 ocasionando epidemias importantes en diferentes países ${ }^{(1,2)}$.

La fiebre Chikungunya es una de las denominadas enfermedades "olvidadas" o "desatendidas", que reemergió debido a factores determinantes como los cambios climáticos, la mutación viral, la urbanización desorganizada con acceso deficiente a fuentes de agua que obliga a almacenarla en recipientes mal tapados o dejados a la intemperie, la diseminación de los vectores y el desplazamiento de las personas en el mundo.

El nombre Chikungunya deriva de una palabra en idioma Makonde del grupo étnico que vive en el sudeste de Tanzania y el norte de Mozambique que significa "aquel que se encorva" o "retorcido", que describe la apariencia inclinada de las personas que sufren la enfermedad, por las artralgias intensas que la caracterizan ${ }^{(2)}$.

El virus ARN Chikungunya (VCHIK) pertenece al género Alfavirus de la familia Togaviridae, que comprende a varios otros que se transmiten principalmente a través de la picadura de artrópodos. Tiene un vector, el mosquito del género Aedes, principalmente Aedes aegypti y Aedes albopictus ${ }^{(2)}$.

Las muertes relacionadas con infección por VCHIK son raras, pero cerca del $30 \%$ de los individuos afectados podrán presentar secuelas a largo plazo que incluyen artralgia y artritis $^{(3)}$.

El virus Chikungunya es transmitido a través de la picadura de los mosquitos del género Aedes, infectados, porque han picado previamente a una persona (con manifestaciones clínicas o no) durante el período de viremia. El período promedio de incubación extrínseca es de 10 días, a partir del cual el mosquito es capaz de transmitir el virus, durante toda su vida, a un individuo susceptible. La enfermedad no se transmite de persona a persona, ni a través de objetos, ni por vía oral, respiratoria ni sexual ${ }^{(1)}$.

La mayoría de las infecciones por VCHIK que ocurren durante el embarazo, el virus no se transmite al feto, aunque existen reportes de abortos espontáneos después de una infección en la madre durante los primeros meses de embarazo. El mayor riesgo de transmisión al recién nacido se produce cuando la mujer tiene viremia en el período intraparto; en este caso la transmisión puede alcanzar al $49 \%$. No hay evidencia de que el virus se transmita a través de la leche materna ${ }^{(4 \cdot 6)}$.

El VCHIK puede afectar a mujeres y hombres de todas las edades. La presentación clínica puede variar con la edad y la presencia de comorbilidades, es sumamente importante distinguirlo del Dengue (Tabla 1). Los neonatos y las personas de edad avanzada y aquellas que presentan comorbilidades pueden desarrollar formas más graves ${ }^{(5,6)}$

Tabla 1: Características diferenciales entre Dengue y Chikungunya ${ }^{(1-3,6,7)}$.

\begin{tabular}{lll}
\hline HALLAZGO & CHIKUNGUNYA & DENGUE \\
\hline FIEBRE & SI, más alta y más prolongada & SI \\
\hline INICIO & Más agudo & Evolución más tórpida \\
\hline EXANTEMA MACULOPAPULAR & Más frecuente & Menos frecuente \\
\hline DIAS EN QUE APARECE EL RASH & Del 1 al 4 día & Del 5 to al 7 mo día \\
\hline DOLOR RETRO-OCULAR & Ausente & Presente \\
ARTARLGIAS CON ARTRITIS & Constante +++ & Ausentes \\
MIALGIAS & Leves & Presentes +++ \\
HIPOTENSION & $\begin{array}{l}\text { A veces cuando la } \\
\text { ingesta es inadecuada }\end{array}$ & $\begin{array}{l}\text { Frecuente por } \\
\text { extravasación }\end{array}$ \\
\hline CHOQUE & Infrecuente & Puede presentarse \\
PLAQUETOPENIA & Temprana pero leve & Tardía pero importante \\
\hline
\end{tabular}

La infección puede ser clínicamente inaparente o puede causar una enfermedad de variada intensidad. Luego de un período de incubación que puede ser de 3 a 7 días, (se han observado casos con un período de incubación de entre 1 hasta 12 días), podrán aparecer las manifestaciones clínicas, aunque entre el $3 \%$ al $28 \%$ de las personas infectadas cursarán de manera asintomática y desarrollarán inmunidad permanente ${ }^{(3-10)}$.

En aquellas personas que desarrollarán síntomas, la enfermedad puede evolucionar de forma aguda, subaguda o crónica.

La enfermedad aguda presenta un inicio súbito de fiebre alta (típicamente mayor a $37,5^{\circ} \mathrm{C}$ ) y dolor articular bilateral, simétrico, de intensidad variable que puede llegar a ser incapacitante. También pueden presentarse exantema maculopapular muy pruriginoso, náuseas, vómitos y conjuntivitis. La fiebre suele durar de 2 a 3 días y asociarse a escalofríos 
y por lo general desaparece con el uso de antipiréticos. No suele observarse variación diurna. Puede ser continua o intermitente, y en general la desaparición de la fiebre no se asocia con empeoramiento de los síntomas clínicos (a diferencia de lo que ocurre en el dengue). Los síntomas articulares suelen afectar más de 1 articulación, con predominio en articulaciones de las manos y muñecas; con menor frecuencia puede involucrar articulaciones mayores como la rodilla, hombro y columna vertebral. Tienden a empeorar en la mañana, y son aliviados por el ejercicio leve y agravados por los movimientos bruscos. Se ha descrito artritis migratoria en cerca del $70 \%$ de los casos. A veces, se asocian a tendosinovitis con inflamación y rigidez en muñecas y tobillos. En algunos casos el dolor es tan intenso que produce incapacidad funcional. El exantema maculopapular suele aparecer entre dos a cinco días después del inicio de la fiebre e incluye tronco y extremidades, aunque también puede afectar palmas, plantas y rostro. La proporción de pacientes que presentan exantema es muy variable entre los estudios; en promedio se presentaría en aproximadamente la mitad de los pacientes. Otras lesiones de la piel incluyen lesiones vesículo-ampollares con descamación (más frecuentes en los niños pequeños), úlceras aftosas y lesiones de tipo vasculítico ${ }^{(2)}$.

En la forma aguda los síntomas suelen remitir en 7 a 10 días y luego, la mayoría de los pacientes sentirá una mejoría en su estado general y una disminución del dolor articular. Sin embargo, posteriormente, pueden reaparecer los síntomas ${ }^{(2,3-12)}$.

La forma sub-aguda, es caracterizada por la afectación articular discapacitante que cede entre el segundo y tercer mes de evolución de la enfermedad, mientras que en la forma crónica la afectación articular persiste por más de tres meses y puede durar, según algunos estudios, hasta 2 a 3 años ${ }^{(2,3)}$.

Los factores de riesgo para la persistencia de los síntomas son la edad avanzada ( $>65$ años), los trastornos articulares preexistentes y la enfermedad aguda más grave. La diabetes mellitus fue la mayor comorbilidad identificada como factor de riesgo para la presencia de artralgia crónica ${ }^{(10)}$.

La frecuencia con que los pacientes presentan síntomas persistentes varía sustancialmente según el estudio y el tiempo transcurrido entre el inicio de los síntomas y el seguimiento. Algunos estudios reportan que entre el $12 \%$ al $18 \%$ de los pacientes tendrán síntomas persistentes a los 18 meses y hasta 2 a 3 años después, mientras que en otros trabajos, la proporción de pacientes con síntomas persistentes a los 10 meses llega al $49 \%{ }^{(10)}$.

En cuanto a las manifestaciones atípicas, alrededor del $0.3 \%-05 \%$ de los afectados pueden presentar éstas manifestaciones que podrían deberse a efectos directos del virus o a la respuesta inmunológica. Dentro de los grupos de riesgo para presentar formas atípicas o graves, se encuentran los recién nacidos de madres virémicas durante el parto o en los últimos 4 días antes del parto, los niños menores de 1 año, los adultos mayores de 65 años, las personas con comorbilidades como diabetes mellitus, hipertensión arterial, insuficiencia renal crónica, enfermedades cardiovasculares, VIH-SIDA, tuberculosis o neoplasias.

Las formas atípicas incluyen afectación neurológica, como meningoencefalitis, encefalopatía, convulsiones, síndrome de Guillain-Barré, síndrome cerebeloso, paresias, parálisis o neuropatía; afectación ocular como un cuadro de neuritis óptica, iridociclitis, retinitis, uveítis; la afectacion cardiovascular comprende la miocarditis, pericarditis, insuficiencia cardíaca, arritmias o la inestabilidad hemodinámica; la afectación dermatológica puede ser hiperpigmentación fotosensible, úlceras, dermatosis vesiculobullosas; en relación a la afectación renal, se puede presentar un cuadro de nefritis, una insuficiencia renal aguda o un síndrome urémico hemolítico.

Otras formas atípicas/ graves son las discrasias sangrantes, neumonía, insuficiencia respiratoria, hepatitis, pancreatitis, síndrome de secreción inadecuada de hormona antidiurética (SIADH), hipoadrenalismo ${ }^{(10)}$.

Para establecer el diagnóstico se pueden utilizar varios métodos y las pruebas serológicas, como la inmunoadsorción enzimática (ELISA), pueden confirmar la presencia de anticuerpos IgM e IgG contra el virus Chikungunya ${ }^{(7)}$. Las muestras recogidas durante la primera semana después de la aparición de los síntomas deben analizarse con métodos serológicos y virológicos (RT-PCR). El virus puede aislarse en la sangre en los primeros días de la infección. Existen diversos métodos de reacción en cadena de la polimerasa con retrotranscriptasa (RT-PCR), pero su sensibilidad es variable. Algunos son idóneos para el diagnóstico clínico. Los productos de RT-PCR de las muestras clínicas también pueden utilizarse en la genotipificación del 
virus, permitiendo comparar muestras de virus de diferentes procedencias geográficas ${ }^{(7)}$.

No existe un tratamiento farmacológico específico. El tratamiento favorece la atención sintomática y el reconocimiento oportuno de las formas atípicas y graves ${ }^{(3-13)}$. Como asimismo en aliviar los síntomas, entre ellos el dolor articular, con antipiréticos, analgésicos óptimos y líquidos. No hay comercializada ninguna vacuna contra el virus Chikungunya $^{(6,9-13)}$. No existe ningún antivírico específico para tratar la fiebre Chikungunya.

Los países afectados con circulación autóctona de este agente infeccioso, actualmente y hasta la semana epidemiológica (SE) 13, son: Estados Unidos de América, Costa Rica, El Salvador, Panamá, Colombia, Venezuela y Caribe, Guyanas, Bahamas, Belice, Trinidad \& Tobago, Jamaica, Brasil, Guatemala, Nicaragua, Honduras, México, Bermuda, Ecuador, Paraguay y Bolivia ${ }^{(8)}$. Uruguay es el país que no ha registrado casos confirmados.

Desde 11/01/2015 (SE 2) hasta la SE 13, se confirmaron un total de 785 casos de Chikungunya, de éstos 395 fueron por laboratorio de referencia y 390 por nexo epidemiológico. La mayoría del número de casos pertenece al Área Metropolitana de Asunción (AMA). Se registraron otros casos confirmados en los departamentos de Paraguarí, Misiones, San Pedro Norte, San Pedro Sur, Presidente Hayes y Cordillera secundarios a los casos del Área metropolitana de Asunción ${ }^{(8)}$.

A continuación se presenta el caso clínico de un Recién nacido con Chikungunya a tener en cuenta en la actualidad cuando se presentan niños en este grupo etario con fiebre de inicio súbito y sin aparentes factores de riesgo materno para sepsis.

\section{CASO CLÍNICO}

Recién nacida de 16 días de vida procedente de Luque, Paraguay, nacida por parto cesárea, de término sin complicaciones. Peso al nacimiento 3,620 gramos, APGAR 8/9 sin factores maternos de riesgo para infección; dada de alta a las 48 hs de vida sin complicaciones. A los 13 días de vida consulta en el servicio de urgencias de un centro público por fiebre y rash, la madre refiere cuadro de $\mathbf{2 4}$ horas de evolución de fiebre y rash generalizado acompañado de irritabilidad transitoria sin otro dato, refiere buena ingesta del pecho materno, es evaluada en el centro asistencial donde se decide su internación, debido al alto riesgo que presenta.

Ingresa con el diagnóstico de Sepsis neonatal tardía probable, se inicia ampicilina mas cefotaxima, se toman análisis de laboratorio al ingreso y llama la atención, leucopenia; se envió además serología para dengue que retorno negativa. En el día 4 de la enfermedad, evoluciona hacia un deterioro progresivo con mayor hipoactividad rechazo alimentario y polipnea, se repiten los análisis laboratoriales y se constata persistencia de leucopenia, y además se aprecia un descenso en el valor de las plaquetas (Tabla 2). No volvió a presentar fiebre, se amplia cobertura antibiótica a ceftazidima más vancomicina.

En el día 5 de enfermedad presenta marcada dificultad respiratoria y se decide una intubación orotraqueal; posteriormente la remiten a un centro de mayor complejidad para cuidados intensivos con el diagnostico de IRAG (Infección respiratoria aguda grave)-Sepsis grave-RNT AEG.

Al ingreso a la unidad de cuidados intensivos neonatales (UCIN), llama la atención al examen físico: edema bipalpebral, de manos y pies; se constata además hipoventilación de ambas bases pulmonares y hepatomegalia, se observó en la radiografía (Figura 1) derrame pleural bilateral además de un infiltrado intersticio alveolar bilateral. En el re-interrogatorio la madre refiere que una hija de 16 años se encuentra con fiebre dolores articulares y rash de igual tiempo de evolución.

Con estos datos se plantean los siguientes diagnósticos:

* RNT-AEG

* Síndrome febril agudo:

- Sospecha de Dengue grave

- Fiebre por Chikungunya a descartar

*Sepsis Grave.

Día 6 de enfermedad presenta fiebre nuevamente, además se constatan crisis convulsiva tónica generalizada por lo que inicia fenobarbital con lo que ceden las crisis. RN muy grave. Se realizó ecocardiografía que informa Hipertensión pulmonar moderada, insuficiencia tricúspide y aortica leve.

Día 7 Paciente delicado pero más estable, sin cambios en la clínica. 
Día 8 de enfermedad Rn estable, se descienden parámetros, en control laboratorial se constató mejoría en relación al recuento de glóbulos blancos pero persiste plaquetopenia.

Tabla 2. Resultados laboratoriales según día de enfermedad.

\begin{tabular}{|c|c|c|c|c|c|c|c|}
\hline $\begin{array}{l}\text { DÍA } \\
\text { LABORATORIO }\end{array}$ & DÍA 2 & DÍA 4 & DÍA 5 & DÍA 6 & DÍA 8 & DÍA 10 & DÍA 15 \\
\hline $\mathrm{Hb} / \mathrm{Hto}$ & $14.5 / 42$ & $13.6 / 41$ & $14,7 / 43$ & $12,2 / 38$ & $11 / 33$ & $11 / 34$ & $10,3 / 32$ \\
\hline G. Blancos & 3.360 & 3.290 & 5.820 & & 10720 & 15.920 & 8.010 \\
\hline Neutrof/Linfoc & $66 / 22 \%$ & $38 / 53 \%$ & $45 / 53 \%$ & & $50 / 46 \%$ & $64 / 34 \%$ & $22 / 73 \%$ \\
\hline Plaquetas & 464.000 & 167.000 & 110.000 & 86.000 & 30.000 & 235.000 & 469.000 \\
\hline PCR & Neg. & Neg. & & & & & \\
\hline Orina & Normal & & & & & & \\
\hline LCR & Normal & & & & & & \\
\hline GAS & & & Ac. Mixta & Normal & Normal & & \\
\hline lonograma & & & Normal & & Normal & Normal & \\
\hline \multirow[t]{3}{*}{ Crasis } & & & Tp 46 & & & & Tp $100 \%$ \\
\hline & & & Fibri 113 & & & & Fibri 292 \\
\hline & & & ТрА 58 & & & & ПТрA 31 \\
\hline Urea/Cr & & & Normal & & & Normal & \\
\hline GOT-GPT-GGT & & & $301 / 63 / 155$ & & $212 / 65 / 85$ & $72 / 47$ & \\
\hline Serogia Ns1 & Neg. & & & Neg. & & & \\
\hline IgM para dengue & Neg. & & & Neg. & & & \\
\hline IgG para dengue & Neg. & & & Neg. & & & \\
\hline PCR CHIKV & & & & & POSTIVO & & \\
\hline IgM para CHIKV & & & & & & POSITIVO & \\
\hline
\end{tabular}

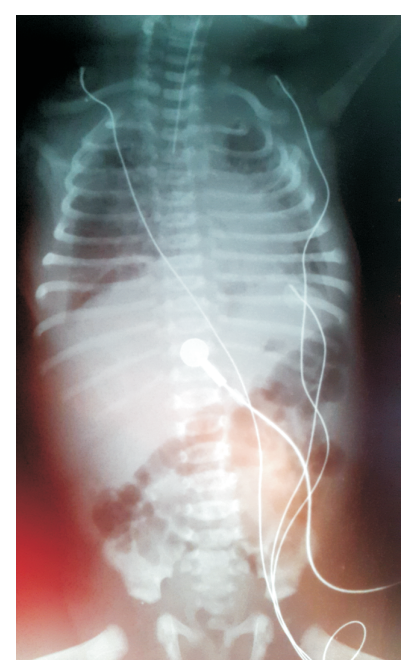

Figura 1. Derrame pleural bilateral e infiltrado intersticio alveolar bilateral.

Día 9 de enfermedad Rn estable en planes de retirar asistencia respiratoria mecánica.

Día 10 de enfermedad se procede a extubación programada exitosa se constató reabsorción total del derrame, ya sin hepatomegalia, con mejoría a nivel de los recuentos de glóbulos blancos, no así en el de las plaquetas que se encontraban en descenso (Tabla 2), completo 7 días de antibióticos, cultivos negativos, se recibe resultado de serología positiva para Chikungunya IgM, es contra referido a centro de origen donde presenta buena evolución, es dado de alta en el día 15 de enfermedad en buenas condiciones y con recuperación hematológica.

\section{DISCUSIÓN}

La población del país es susceptible de padecer esta enfermedad, en general las formas graves se presentan en Recién Nacidos, Adultos mayores y pacientes con comorbilidad, los síntomas en los niños son muy parecidos a los del adulto $^{(9)}$ Ocasionalmente, las complicaciones son los trastornos neurológicos como la meningoencefalitis que puede ser letal, también pueden presentarse miocarditis y epidermólisis extensa. La transmisión madre-hijo, provoca una infección neonatal de CHIKV, y se observa cerca del final del embarazo de madres en la fase febril virémica. (Tasa de transmisión vertical $49-50 \%)^{(4,10)}$. Los síntomas suelen aparecer a partir del $3^{\text {er }}$ día de nacido y hasta el $7^{\mathrm{mo}}$ día, con una media de 4 días, pueden iniciar como en nuestro caso con fiebre, rechazo alimentario, irritabilidad, edema distal, además existen diversas manifestaciones de la piel (erupción maculopapular, epidermólisis, vesículas o bullas), o en casos más graves Crisis convulsivas, Meningoencefalitis, Miocarditis o Anormalidades ecocardiográficas ${ }^{(4-6,10)}$. $\mathrm{Si}$ se consideran estas manifestaciones es necesario insistir con el interrogatorio, en el afán de establecer nexos epidemiológicos para la orientación diagnóstica cuando se tienen casos en donde el diagnóstico no está claro al inicio.

En un estudio presentado en República Dominicana en el 2014 sobre 79 casos se vio que 74 madres eran sintomáticas al momento del parto, $50 \%$ de estos niños desarrollaron una forma grave de $\mathrm{CHIKV}$ con una tasa de secuelas neurológicas de un $50 \%$. Las principales complicaciones descritas asociadas al CHIKV en neonatos en este estudio fueron las siguientes: falla respiratoria, descompensación cardiovascular, meningoencefalitis, hepatitis aguda, manifestaciones cutáneas severas (descamación y lesiones bullosas) ${ }^{(2,3,8)}$.

El caso clínico presentado escapa al de una transmisión materna por el tiempo en el que empezaron los síntomas, se trata más bien de un caso adquirido de Chikungunya pues los síntomas empezaron en el día 13 de vida. La madre tiene 
serología negativa para Chikungunya. No se registran reportes de casos clínicos de CHIKV adquiridos en recién nacidos excepto los reportados por el trabajo hecho en República Dominicana ${ }^{(9)}$

Se considera el presente caso como GRAVE ATÍPICO de Chikungunya. Un caso es atípico cuando el paciente requiere mantenimiento de por lo menos una de las funciones vitales con una prueba confirmada de Chikungunya y que desarrolla otros síntomas más que la fiebre y el $\operatorname{rash}^{(9,6,13)}$, en el caso presentado los síntomas fueron afectación respiratoria grave que requirió ARM y

\section{REFERENCIAS}

1. Organización Panamericana de la Salud. Alerta epidemiológica: fiebre por chikungunya. 9 diciembre 2013 [Internet]. Washington, DC: OPS; 2013 [citado 2015 Ene 21]. Disponible en: http://www.paho.org/hq/index.php?o ption=com_docman\&task=doc_view\&gid=23807\&Itemid

2. Ministerio de Salud Pública. Guía de manejo clínico para la infección por el virus Chikungunya (CHIKV). Santo Domingo, República Dominicana: Ministerio de Salud Pública,. 2014.

3. PAHO/WHO. Preparedness and response for Chikungunya virus introduction in the Americas [Internet]. Washington; PAHO/WHO; 2011 [Citado 2015 Ene 10] Disponible en: http://www.paho.org/hq/index. php?option=com_docman\&task=doc_download\&gid=16 984\&Itemid=\&lang=en.

4. Lenglet Y, Barau G, Robillard PY, Randrianaivo H, Michault A, Bouveret A, Gérardin P, Boumahni B, Touret Y, Kauffmann E, Schuffenecker I, Gabriele M, Fourmaintraux A. Chikungunya infection in pregnancy: Evidence for intrauterine infection in pregnant women and vertical transmission in the parturient. Survey of the Reunion Island outbreak. J Gynecol Obstet Biol Reprod (Paris). 2006;35(6):578-83.

5. Ramful D, Carbonnier M, Pasquet M, Bouhmani B, Ghazouani J, Noormahomed T, Beullier G, Attali T, Samperiz S, Fourmaintraux A, Alessandri JL. Mother to child transmission of chikunguya virus infection. Pediatr Infect Dis J. 2007;26(9):811-5.

6. Ministerio de Salud Pública y Bienestar Social. Guía para el manejo clínico de la enfermedad producida por el virus de la Chikungunya. Asunción: OPS/OMS; 2015. neurológica (convulsiones), el manejo como describe la literatura hasta el momento es de sostén para las complicaciones.

Es necesario tener presente la definición de casos, por la situación epidemiológica; el Ministerio de Salud Pública y Bienestar Social del Paraguay dispone de la Guía de Manejo Clínico muy práctico para el efecto ${ }^{(6)}$. También es importante el seguimiento posterior de este tipo de pacientes ya que existen formas crónicas reportadas en adultos pero debido a lo atípico del caso, se desconocen las secuelas eventuales y más todavía, en el caso de los recién nacidos.
7. WHO. Chikungunya. Nota descriptiva $N^{\circ} 327$ Octubre de 2014 [Interntet]. WHO; 2014 [Citado 2015 Ene 20] Disponible en: http://www.who.int/mediacentre/factshee ts/fs327/es/

8. Ministerio de Salud Pública y Bienestar Social. Dirección General de Vigilancia de la Salud. Semana Epidemiológica 13. Boletín Epidemiológico. 2015;13 (13 de abril de 2015).

9. Pimentel R, Skewes-Ramm, Moya J. Chikungunya en la República Dominicana: lecciones aprendidas en los primeros seis meses. Rev Panam Salud Pública. 2014;36(5):336-41.

10. Atypical Chikungunya virus infection: clinical manifestation ,mortality and risk factor for severe disease during 2005-2006 outbreak on Reunión. Epidemiology and Infection. 2009;137(4):534-41.

11. Moya J, Pimentel R, Puello J. Chikungunya: un reto para los servicios de salud de la República Dominicana. Rev Panam Salud Publica. 2014;36(5):331-35.

12. OPS/OMS. Chikungunya [Internet]. Washington: OPS [citado 2015 Ene 21]. Disponible en: http://www.paho.org/ hq/index.php?option $=$ com_topics\&view $=$ article\&id $=343$ \&Itemid=40931\&lang=es

13.WHO. Guidelines on clinical management of Chikungunya fever [Internet]. 2008 [citado 2015 Ene 21]. Disponible en: http://www.wpro.who.int/mvp/topics/ntd /Clinical_Mgnt_Chikungunya_WHO_SEARO.pdf 